\title{
OPTIMAL FEEDBACK CONTROL OF BATCH REACTORS WITH A STATE INEQUALITY CONSTRAINT AND FREE TERMINAL TIME
}

\author{
SRINIVAS PALANKI, ${ }^{\dagger}$ COSTAS KRAVARIS and HENRY Y, WANG \\ Department of Chemical Engineering, The University of Michigan, Ann Arbor, MI 48109, U.S.A.
}

(First received 23 December 1992; accepted in revised form 19 May 1993)

\begin{abstract}
In this paper we derive optimal state feedback laws for end-point optimization of a dynamic system where the final time is free and the system has a scalar inequality constraint. The existence of a singular region as well as the nature of the state feedback law (static or dynamic) is completely characterized in terms of the system dynamics. Explicit synthesis formulae for the state feedback laws are presented. Once the state feedback laws for end-point optimization have been derived, issues on how these laws can be implemented as part of a closed-loop scheme are discussed. As illustrative examples of application of the proposed methodology, several end-point optimization problems in batch chemical reactors are considered.
\end{abstract}

\section{INTRODUCTION}

Batch and semi-batch processes are of great importance to the chemical industry. A wide variety of speciality chemicals such as antibiotics and polymers are produced in batch reactors. Since batch reactors produce low-volume, high value products, optimal operation is very important. In a previous paper (Palanki et al., 1993), we had synthesized optimal feedback laws for end-point optimization of batch processes with fixed final time. In this paper we will study the general problem of synthesizing optimal state feedback laws which guarantee optimality when the system has a state inequality constraint and the final time of the batch is left free.

State inequality constraints are usually physical constraints to the system. For example, in a semibatch reactor one can feed only a finite amount of substrate due to a volume constraint on the reactor. Similarly, one may not want to operate a reactor beyond a certain maximum temperature for safety reasons. In a fed-batch bioreactor there could be constraints on the cell mass concentration (beyond which oxygen transfer is limited) or the substrate concentration (beyond which undesirable side reactions occur). Due to batch-to-batch variation in yield, the final time may not be fixed a priori. The reactor operation is stopped when the optimum yield is achieved. Thus, it is important to consider yield optimization problems with state inequality constraints and free terminal time. The purpose of this paper is to develop state feedback laws for such optimization problems.

†Present address: Department of Chemical Engineering Florida A \& M University/Florida State University, College of Engineering, Tallahassee, FL 32316, U.S.A. Author to whom correspondence should be addressed.

\section{FORMULATION OF THE END-POIN'T OPTIMIZATION PRORLEM: THE ClASSICAL OPTIMAL CONTROL PERSPECTIVE}

The end-point optimization problem can be mathematically formulated as follows:

Minimize the performance index,

$$
J=\phi\left(x\left(t_{f}\right), t_{f}\right)
$$

subject to the dynamics

$$
\begin{gathered}
\dot{x}=f(x)+g(x) u, \quad 0 \leqslant t \leqslant t_{f} \quad u_{\min } \leqslant u \leqslant u_{\max } \\
x(0)=x_{0}
\end{gathered}
$$

and the scalar state inequality constraint:

$$
C(x) \leqslant 0 .
$$

In this formulation $u$ is the scalar manipulated input bounded by $u_{\min }$ and $u_{\max }, x$ is the $n$-vector of states, $t_{f}$ is the free final time, $f(x)$ and $g(x)$ are smooth vector functions and $\phi(x)$ is a smooth scalar function such that

$$
\begin{aligned}
g(x) & \neq\left[\begin{array}{lll}
0 & 0 & \ldots
\end{array}\right] \\
\frac{\partial \phi}{\partial x} & \neq\left[\begin{array}{lll}
0 & 0 & \ldots
\end{array}\right]
\end{aligned}
$$

for all $x$.

The part of the optimal solution where the state constraint is hit $(C(x)=0)$ is called the constrained arc or the boundary arc. The part of the solution where $C(x)<0$ is called the unconstrained arc or the interior arc.

Necessary conditions for optimality on an uncon strained arc

By Pontryagin's Principle, the minimization problem (1) is equivalent to minimizing the Hamiltonian:

$$
H(x, \lambda, u)=\lambda^{T} f(x)+\lambda^{T} g(x) u
$$


where $\lambda$ is the solution of

$$
\begin{aligned}
\lambda^{T} & =-\lambda^{T} \frac{\partial f}{\partial x}(x)-\lambda^{T} \frac{\partial g}{\partial x}(x) u \\
\lambda\left(t_{f}\right) & =\left.\frac{\partial \phi\left(x, t_{f}\right)}{\partial x}\right|_{t=t_{s}} .
\end{aligned}
$$

The coefficient of $u$ in eq. (5), $\lambda^{T} g(x)$, is called the switching function. When the switching function is negative, $u=u_{\max }$ and when the switching function is positive, $u=u_{\min }$. The region where the switching function vanishes is called the singular region. In the singular rcgion,

$$
\lambda^{T} g(x)=0 .
$$

Therefore its successive time derivatives are also zero. Thus, we have:

$$
\begin{gathered}
\frac{\mathrm{d}}{\mathrm{d} t}\left(\lambda^{T} g(x)\right)=0 \\
\frac{\mathrm{d}^{2}}{\mathrm{~d} t^{2}}\left(\lambda^{T} g(x)\right)=0 .
\end{gathered}
$$

This sequence of differentiations is performed until $u$ appears explicitly. The resulting expression can then be solved for $u$ in terms of $x$ and $\lambda$. Furthermore, since the final time is free (Bryson and Ho, 1975)

$$
\left(\frac{\partial \phi}{\partial t}+H\right)_{r=t s}=0
$$

When the objective function $\phi$ is not an explicit function of the final time $t_{f}$, we obtain

$$
(H)_{t=t_{f}}=0 \text {. }
$$

Furthermore, since the functions $f$ and $g$ are not explicit functions of time, the Hamiltonian $\boldsymbol{H}$ is constant on the optimal trajectory (Bryson and Ho, 1975). Thus,

$$
H=(H)_{t=t_{s}}=0 .
$$

Since, $\lambda^{T} g(x)=0$ in the singular region, we have, from equation (11),

$$
\lambda^{T} f(x)=0
$$

in the singular region. This can be used as an extra first-order necessary condition for optimality when the objective function $\phi$ is not an explicit function of the final time $t_{f}$.

Necessary conditions for optimality on a constrained arc

On the constrained arc

$$
C(x)=0 .
$$

The Hamiltonian $H$ is defined as

$$
H=\lambda^{T} f(x)+\lambda^{T} g(x) u+\mu(t) C(x)
$$

where

$$
\mu(t) \geqslant 0
$$

and the adjoint states are defined as (Bryson et al., 1963)

$$
\frac{\mathrm{d} \lambda^{T}}{\mathrm{~d} t}=-\lambda^{T} \frac{\partial f(x)}{\partial x}-\lambda^{T} \frac{\partial g(x)}{\partial x} u-\mu(t)\left[\frac{\partial C(x)}{\partial x}\right] .
$$

On the constraint boundary, $\mu(t)$ is so chosen that

$$
\frac{\partial H}{\partial u}=\lambda^{T} g(x)=0 .
$$

Thus, the boundary arc is a singular arc in the sense of the minimum principle (Maurer, 1977).

\section{Solution of optimization problem}

There are $2 n+2$ equations [ $n$ state equations, $n$ adjoint equations, eq. (9), and eq. (17)] and $2 n+2$ variables $[n$ state variables, $n$ adjoint variables, the final time $t_{f}$ and the parameter $\left.\mu(t)\right]$. However, the boundary conditions for the state and adjoint equations are split, i.e. the initial conditions of the state equations and the terminal conditions of the adjoint equations are known. Thus, we have a two point boundary value problem which can be computationally quite expensive to solve. Furthermore, in this formulation, the numerical problem has to be solved separately every time there is a change in initial conditions or model parameters. The optimal solution has to be implemented in an open-loop fashion and so uncertainties and disturbances to the process are not attenuated. These problems can be alleviated if the optimal solution is derived as a state feedback law.

\section{END-POINT OPTIMIZATION OF FREE TIME PROBLEMS A GEOMETRIC PERSPECTIVE}

The previous sub-section gave an overview of the classical optimal control formulation. In the present subsection it will be seen that geometric tools can be used to obtain a more concrete and transparent representation of the necessary conditions for optimality. These results will be used to develop a general framework for end-point optimal state feedback synthesis.

Throughout this section, we will make the standing assumption that $g(x), a d_{f}^{1} g(x), \ldots, a d_{f}^{n-1} g(x)$ are linearly independent almost everywhere in $x$, where $\operatorname{ad}_{f}^{k} g(x)$ is the system Lie bracket of order $k$. This assumption guarantees local controllability of the system almost everywhere (Hunt, 1982) and is equivalent to

$$
\operatorname{det}\left[g(x) \vdots a d_{f}^{1} g(x) \vdots \ldots \vdots a d_{f}^{n-1} g(x)\right] \not \equiv 0 .
$$

\section{Optimal state feedback laws on unconstrained arcs}

Definition 1 (Krener, 1977; Palanki et al., 1993): The system governed by eq. (2) has a finite degree of singularity $s$ if

$$
\begin{gathered}
{\left[g, a d_{f}^{v} g\right](x) \in \operatorname{span}\left\{g(x), a d d_{f}^{1} g(x), \ldots, a d_{f}^{v} g(x)\right\}} \\
\text { for all } v \leqslant s-1 \\
{\left[g, a d_{f}^{s} g\right](x) \notin \operatorname{span}\left\{g(x), a d_{f}^{1} g(x), \ldots, a d_{f}^{z} g(x)\right\} .}
\end{gathered}
$$


The system governed by equation (2) has an infinite degree of singularity $(s=\infty)$ if

$$
\begin{array}{r}
{\left[g, a d_{f}^{v} g\right](x) \in \operatorname{span}\left\{g(x), a d_{f}^{1} g(x), \ldots, a d_{f}^{v} g(x)\right\}} \\
\text { for all } v \geqslant 0 .
\end{array}
$$

Since $g(x), a d_{f}^{1} g(x), \ldots, a d_{f}^{n-1} g(x)$ are assumed to be linearly independent, it follows from the definition that $1 \leqslant s \leqslant(n-2)$ or $s=\infty$.

The following proposition is a rather straightforward consequence of known results (see Gabasov and Kirillova, 1972).

ries corresponding to a singular extremal evolve on the surface given by

$$
\begin{aligned}
& S(x)= \\
& \quad \operatorname{det}\left[f(x) \vdots g(x) \vdots a d_{f}^{1} g(x) \vdots \ldots \vdots a d_{f}^{n-2} g(x)\right]=0 .
\end{aligned}
$$

(ii) If $\mathrm{f}(\mathrm{x})$ is linearly dependent on $\mathrm{g}(\mathrm{x})$, $\operatorname{ad}_{f} \mathrm{~g}(\mathrm{x}), \ldots, \mathrm{ad}_{\mathrm{f}}^{-2} \mathrm{~g}(\mathrm{x})$ or the performance index depends explicitly on the free final time, then the optimal static state feedback is given by

$$
u=-\frac{\operatorname{det}\left[g(x) \vdots a d_{f}^{1} g(x) \vdots \ldots \vdots a d_{f}^{n-1} g(x)\right]}{\operatorname{det}\left[g(x) \vdots a d_{f}^{1} g(x) \vdots \ldots \vdots a d_{f}^{n-2} g(x) \vdots\left[g, a d_{f}^{n-2} g\right](x)\right]} .
$$

Proposition 1: Consider a system governed by eq. (2) with finite degree of singularity $\mathrm{s}$. Then $\mathrm{s}$ is odd and the first-order necessary conditions for optimality for a singular extremal can be written as follows:

$$
\begin{gathered}
\lambda^{\top} a d_{f}^{k} g(x)=0 \quad k=0,1,2, \ldots, s \\
\lambda^{T} a d_{f}^{s+1} g(x)+\lambda^{T}\left[g, a d_{f}^{s} g\right](x) u=0 .
\end{gathered}
$$

\section{Proof: See Appendix B.}

Theorem 3: Consider a system of the form of eq. (2) with degree of singularity $\mathrm{s}=\mathrm{n}-3$. (i) $\mathrm{I} \mathrm{f}(\mathrm{x})$ is linearly independent of $\mathrm{g}(\mathrm{x}), \mathrm{ad}_{\mathrm{f}}^{1} \mathrm{~g}(\mathrm{x}), \ldots, \mathrm{ad}_{\mathrm{f}}^{-2} \mathrm{~g}(\mathrm{x})$ and the performance index does not depend explicitly on the free final time, then the optimal static state feedback is given by

$$
u=-\frac{\operatorname{det}\left[f(x) \vdots g(x) \vdots a d_{f}^{1} g(x) \vdots \ldots \vdots a d_{f}^{n-2} g(x)\right]}{\operatorname{det}\left[f(x) \vdots g(x) ; a d_{f}^{1} g(x) \vdots \ldots \ldots d_{f}^{n-3} g(x) \vdots\left[g, a d_{f}^{n-3} g\right](x)\right]}
$$

Furthermore, the second-order necessary condition for optimality in the singular region takes the form

$$
(-1)^{\frac{s+1}{2}} \lambda^{T}\left[g, a d^{s}, g\right](x) \geqslant 0
$$

If eq. (2) has an infinite degree of singularity, then the first-order necessary conditions for optimality take the form $\lambda^{\mathrm{T}} \mathrm{ad}_{\mathrm{i}}^{\mathrm{g}} \mathrm{g}(\mathrm{x})=0$ for all $\mathrm{v} \geqslant 0$.

If $\phi$ is independent of the final time $t_{f}$, then an additional necessary condition for optimality in the singular region is

$$
\lambda^{T} f(x)=0
$$

Theorem 1: Consider a system of the form of eq. (2) with degree of singularity $\mathrm{s}=\infty . \mathrm{I} f \mathrm{f}(\mathrm{x})$ is linearly dependent on $\mathrm{g}(\mathrm{x})$, ad $\mathrm{g}(\mathrm{x}), \ldots, \operatorname{ad}_{\mathrm{f}}^{\mathbf{n}}-\mathbf{g}(\mathrm{x})$ almost everywhere or the performance index depends explicitly on the free final time, then the state trajectories corresponding to a singular extremal evolve on the following surface:

$$
S(x)=\operatorname{det}\left[g(x) \vdots a d_{f}^{1} g(x) \vdots \ldots \vdots a d_{f}^{n-1} g(x)\right]=0 .
$$

Proof: See Appendix A.

Theorem 2: Consider a system of the form of eq. (2) with degree of singularity $\mathrm{s}=\mathrm{n}-2$. (i) If $\mathrm{f}(\mathrm{x})$ is linearly independent of $\mathrm{g}(\mathrm{x}), \operatorname{ad}_{\mathrm{f}}^{1} \mathrm{~g}(\mathrm{x}), \ldots, \mathrm{ad}_{\mathrm{f}}^{\mathrm{n}-2} \mathrm{~g}(\mathrm{x})$ almost everywhere and the performance index does not depend explicitly on the free final time, then the state trajecto- (ii) If $\mathrm{f}(\mathrm{x})$ is linearly dependent on $\mathrm{g}(\mathrm{x}), \operatorname{ad}_{\mathrm{f}}^{1} \mathrm{~g}(\mathrm{x})$, $\ldots ; \operatorname{ad}^{n-2} \mathrm{~g}(\mathrm{x})$ or the performance index depends explicitly on the free final time, then the optimal dynamic state feedback is given by

$$
\Phi\left(x, u, \frac{\mathrm{d} u}{\mathrm{~d} t}\right)=0
$$

where $\Phi$ is an algebraic function. This is obtained by setting the determinant of

$$
\begin{aligned}
& g(x) \\
& a d_{f}^{1} g(x) \\
& \vdots \\
& a d_{f}^{n-3} g(x) \\
& a d_{f}^{n-2} g(x)+\left[g, a d_{f}^{n-3} g\right](x) u(t) \\
& a d_{f}^{n-1} g(x)+\left[g, a d_{f}^{H-2} g\right](x) u(t) \\
& +\left(I \frac{\mathrm{d}}{\mathrm{d} t}-\frac{\partial f(x)}{\partial x}-\frac{\partial g(x)}{\partial x} u(t)\right)\left(\left[g, a d_{f}^{n-3} g\right](x) u(t)\right)
\end{aligned}
$$

equal to zero.

In the above equations, $I$ is the identity matrix and $\frac{d(.)}{d t}$ is the total time derivative operator,

$$
\frac{\mathrm{d}(.)}{\mathrm{d} t}=[f(x)+g(x) u(t)] \frac{\partial(.)}{\partial x}+\frac{\partial(.)}{\partial t} .
$$

Theorem 4: Consider a system of the form of eq. (2) with degree of singularity $\mathrm{s} \leqslant \mathrm{n}-4$. (i) If $\mathrm{f}(\mathrm{x})$ is linearly 
independent of $\mathrm{g}(\mathrm{x}), \operatorname{ad}_{\mathrm{f}}^{1} \mathrm{~g}(\mathrm{x}), \ldots, \operatorname{ad}_{\mathrm{f}}^{\mathrm{n}}-\mathbf{2} \mathrm{g}(\mathrm{x})$ and the performance index does not depend explicitly on the free final time, then the optimal dynamic state feedback is given by

$$
\Phi\left(x, u, \frac{\mathrm{d} u}{\mathrm{~d} t}, \ldots, \frac{\mathrm{d}^{n-s-3} u}{\mathrm{~d} t^{n-s-3}}\right)=0
$$

where $\Phi$ is an algebraic function. This is obtained by setting the determinant of

$$
\begin{aligned}
& f(x) \\
& g(x) \\
& a d_{f}^{1} g(x) \\
& \vdots \\
& a d_{f}^{s-1} g(x) \\
& a d_{f}^{s} g(x)+\left[g, a d_{f}^{s-1} g\right](x) u(t) \\
& a d_{f}^{s+1} g(x)+\left[g, a d_{f}^{s} g\right](x) u(t) \\
& +\left(I \frac{\mathrm{d}}{\mathrm{d} t}-\frac{\partial f(x)}{\partial x}-\frac{\partial g(x)}{\partial x} u(t)\right)\left(\left[g, a d_{f}^{s-1} g\right](x) u(t)\right) \\
& \vdots \\
& a d_{f}^{n-2} g(x)+\sum_{k=0}^{n-s-3}\left(I \frac{\mathrm{d}}{\mathrm{d} t}-\frac{\partial f(x)}{\partial x}\right. \\
& \left.\quad-\frac{\partial g(x)}{\partial x} u(t)\right)^{k}\left(\left[g, a d_{f}^{n-k-3} g\right](x) u(t)\right)
\end{aligned}
$$

equal to zero.

(ii) If $\mathrm{f}(\mathrm{x})$ is linearly dependent on $\mathrm{g}(\mathrm{x})$, $\operatorname{ad}_{\mathrm{f}} \mathrm{g}(\mathrm{x}), \ldots, \mathrm{ad}_{\mathrm{f}}^{\mathrm{n}-2} \mathrm{~g}(\mathrm{x})$ or the performance index depends explicitly on the free final time, then the optimal dynamic state feedback is given by

$$
\Phi\left(x, u, \frac{\mathrm{d} u}{\mathrm{~d} t}, \ldots, \frac{\mathrm{d}^{n-s-2} u}{\mathrm{~d} t^{n-s-2}}\right)=0
$$

where $\Phi$ is an algebraic function. This is obtained by setting the determinant of

$$
\begin{aligned}
& g(x) \\
& a d_{f}^{1} g(x) \\
& \vdots \\
& a d_{f}^{s} g(x) \\
& a d_{f}^{s+1} g(x)+\left[g, a d_{f}^{s} g\right](x) u(t) \\
& a d_{f}^{s+2} g(x)+\left[g, a d_{f}^{s+1} g\right](x) u(t) \\
& \quad+\left(I \frac{\mathrm{d}}{\mathrm{d} t}-\frac{\partial f(x)}{\partial x}-\frac{\partial g(x)}{\partial x} u(t)\right)\left(\left[g, a d_{f}^{s} g\right](x) u(t)\right) \\
& \quad \vdots \quad a d_{f}^{n-1} g(x)+\sum_{k=0}^{n-s-2}\left(I \frac{\mathrm{d}}{\mathrm{d} t}-\frac{\partial f(x)}{\partial x}\right. \\
& \left.\quad-\frac{\partial g(x)}{\partial x} u(t)\right)^{k}\left(\left[g, a d_{f}^{n-k-2} g\right](x) u(t)\right)
\end{aligned}
$$

equal to zero.
Proof: See Appendix D.

Optimal state feedback laws on constrained arcs

On the constrained arc, the optimization problem is reduced to the following regulation problem:

Regulate the output

$$
y=C(x)
$$

with zero set point subject to dynamics:

$$
\dot{x}=f(x)+g(x) u .
$$

At the point where the system enters or leaves a constrained arc, a feasible unconstrained arc passing through this point must satisfy certain tangency conditions, namely, this arc must have zero values of the state variable constraint and all its time derivatives that do not involve the control variable (Speyer and Bryson, 1968). Thus, if the relative order of the system (33) is $r$, then at the entry point, exit point and on the constrained arc, the following tangency conditions must be satisfied:

$$
\begin{gathered}
C(x)=L_{f}^{0} C(x)=0 \\
\frac{\mathrm{d} C(x)}{\mathrm{d} t}=L_{f}^{1} C(x)=0 \\
\vdots \\
\frac{\mathrm{d}^{r-1} C(x)}{\mathrm{d} t^{r-1}}=L_{f}^{r-1} C(x)=0 .
\end{gathered}
$$

If the system possesses a finite relative order, a state feedback for this regulation problem can be found by standard techniques. For instance (Kravaris and Chung, 1987) the state transformation,

$$
u=\psi(x, v)=\frac{v-\sum_{k=0}^{r} \beta_{k} L_{f}^{k} C(x)}{\beta_{r} L_{g} L_{f}^{r-1} C(x)}
$$

transforms the system (33) into an input/output linear system of the form

$$
\sum_{k=0}^{r} \beta_{k} \frac{d^{k} y}{d t^{k}}=v
$$

where $\beta_{0}, \beta_{1}, \ldots, \beta_{r}$ are completely arbitrary. When the poles of the $v-y$ system are appropriately placed "far left" in the complex plane, one can use an external linear controller to force the system output $y(t)$ to zero.

\section{Implementation}

In the previous section, state feedback laws have been derived for the unconstrained as well as the constrained regions. To implement the optimal state feedback laws as a closed-loop scheme, the sequence in which singular, nonsingular and constrained arcs appear should be known a priori. Furthermore, the switching times between singular, non-singular and constrained ares is required. The switch to a constrained arc from an unconstrained arc occurs when the system reaches the constraint boundary. The switch to a singular arc can he calculated analytically 
for a restricted class of systems [for example, for systems where a switching surface such as eqs (24) and (25) can be calculated]. In most practical situations, these have to be computed numerically. From the off-line solution of the first-order necessary conditions of optimality, the switching times and the values of the system states at the junctions between singular and non-singular regions are known. This information can be used to set up on-line criteria for the onset of the singular region in terms of the system states (Modak, 1988). Alternatively, one can use the a priori calculated switching times determined from the off-line analysis. When the state feedback law is dynamic, we
The forward reaction is second order with respect to the concentration of species A while the reverse reaction is first order with respect to the concentration of species B. It is desired to find the optimal temperature profile to minimize the following objective function:

$$
J=-\left(C_{B}-\varepsilon t_{f}\right)
$$

There is a constraint on the maximum allowable reactor temperature

$$
T-T_{\max } \leqslant 0 .
$$

The manipulated input is the cooling rate of the reactor. The mass and energy balance for this system are as follows.

$$
\frac{\mathrm{d}}{\mathrm{d} t}\left[\begin{array}{c}
C_{A} \\
T
\end{array}\right]=\left[\begin{array}{c}
-k_{1}(T) C_{A}^{2}+k_{2}(T)\left(C_{A 0}-C_{A}\right) \\
\left(\frac{-\Delta H}{\rho C_{p}}\right)\left(k_{1}(T) C_{A}^{2}-k_{2}(T) C_{A 0}+k_{2}(T) C_{A}\right)
\end{array}\right]+\left[\begin{array}{c}
0 \\
\frac{T_{c}-T}{\rho C_{p}}
\end{array}\right] u
$$

also need initial conditions for the dynamic state feedback law. These initial conditions have to be set up in a similar fashion as the switching times. If the model parameters are known a priori, the feedback laws derived in the previous sections can be used in conjunction with state estimators. Such an implementation can attenuate errors in the initial conditions where

$$
\begin{aligned}
& k_{1}=k_{10} \exp \left[\frac{-E_{1}}{R T}\right] \\
& k_{2}=k_{20} \exp \left[\frac{-E_{2}}{R T}\right] .
\end{aligned}
$$

This system is of the form (2) with

$$
x=\left[\begin{array}{c}
C_{A} \\
T
\end{array}\right], \quad f(x)=\left[\begin{array}{c}
-k_{1}(T) C_{A}^{2}+k_{2}(T)\left(C_{A 0}-C_{A}\right) \\
\left(\frac{-\Delta H}{\rho C_{p}}\right)\left(k_{1}(T) C_{A}^{2}-k_{2}(T) C_{A O}+k_{2}(T) C_{A}\right)
\end{array}\right], \quad g(x)=\left[\begin{array}{c}
0 \\
\frac{T_{c}-T}{\rho C_{p}}
\end{array}\right] .
$$

and process disturbances. If some of the model parameters are not accurately known a priori, these need to be estimated on-line. In this situation, a parameter estimation algorithm is also required.

If one uses a priori determined switching times and model parameters, a successful feedback implementation of the optimal feedback law depends on how sensitive the final performance index is to errors in the switching times. In the absence of rigorous robustness proofs, one resorts to numerical simulations to define a region in the parameter space where the feedback implementation is successful.

The final time can be calculated on-line as follows. Using eqs (5) and (6), it can be shown that

$$
(H)_{t=t_{f}}=\left(\frac{\partial \phi}{\partial x} f(x)+\frac{\partial \phi}{\partial x} g(x) u\right)_{t=1, j}=\left(\frac{\partial \phi}{\partial x} \dot{x}\right)_{t=t_{f}} .
$$

Substituting the right-hand side of the above equation in (9), we obtain

$$
\left(\frac{\partial \phi}{\partial t}+H\right)_{t=t_{\rho}}=\left(\frac{\partial \phi}{\partial t}+\frac{\partial \phi}{\partial x} \dot{x}\right)_{t=t_{s}}=\left(\frac{\mathrm{d} \phi}{\mathrm{d} t}\right)_{t=t_{s}}=0 .
$$

The stopping condition is obtained at the point where the performance index reaches its optimum value.

\section{ILLUSTRATIVE EXAMPLES}

Example 1: The following exothermic reversible reaction is taking place in a batch reactor:

$$
A \rightleftharpoons B \text {. }
$$

It can be easily shown that $n=2, s=\infty$ and $g(x)$ and $a d_{f}^{1} g(x)$ are linearly independent almost everywhere in $x$.

\section{Unconstrained arc}

Equation (38) depencis explicitly on the final time and so eq. (24) is valid in the singular region. Using eq. (24) we obtain

$$
\begin{aligned}
S\left(C_{A}, T\right)= & \operatorname{det}\left[g(x) \vdots a d_{f}^{1} g(x)\right]=E_{1} k_{1}(T) C_{A}^{2} \\
& +E_{2} k_{2}(T) C_{A}-E_{2} k_{2}(T) C_{A 0}=0 .
\end{aligned}
$$

Thus, the optimization problem reduces to regulating the function $S\left(C_{A}, T\right)$ to zero where the system states $C_{A}$ and $T$ are described by eq. (40). It can be easily verified that the relative order for this regulation problem is unity. Thus, an appropriate nonlinear feedback law for this problem is (see Kravaris and Chung, 1987)

$$
u=-\frac{\left[\frac{\partial S}{\partial x} f(x)+\beta_{1} S\right]}{\frac{\partial S}{\partial x} g(x)}
$$

where $x, f(x)$ and $g(x)$ are given by eq. (42), the expression for $S$ is given by eq. (43) and $\beta_{1}$ is an adjustable parameter. Substituting for $x, f(x), g(x)$ and 
$S$, we obtain

$u=-\frac{\frac{\partial S}{\partial C_{A}}\left(-k_{1} C_{A}^{2}+k_{2}\left(C_{A 0}-C_{A}\right)\right)+\frac{\partial S}{\partial T}\left(\left(\frac{-\Delta H}{\rho C_{p}}\right)\left(k_{1} C_{A}^{2}-k_{2} C_{A 0}+k_{2} C_{A}\right)\right)+\beta_{1} S}{\frac{\partial S}{\partial T}\left(\frac{T_{c}-T}{\rho C_{p}}\right)}$

\section{Constrained arc}

On the constraint boundary the control objective is to keep the system at $C(T)=T-T_{\max }=0$. It can be easily verified that for this system the relative order is unity. Thus, we derive a nonlinear feedback law similar to eq. (44) to obtain

$u=-\frac{\partial T\left(\left(\begin{array}{c}-\Delta H \\ \rho C_{p}\end{array}\right)\left(k_{1} C_{A}^{2} \quad k_{2} C_{A 0}+k_{2} C_{A}\right)\right)+\beta_{2} C}{\frac{\partial C}{\partial T}\left(\frac{T_{c}-T}{\rho C_{p}}\right)}$

where $B_{2}$ is an adjustable parameter.

Figure 1 shows the optimal temperature profile for the system parameters given in Table 1 and the initial conditions given in Table 2 . It was found through numerical simulations that $\beta_{1}=1$ and $\beta_{2}=1$ were appropriate for this system. Figure 2 shows the optimal cooling rate of the reactor. Since the reactant is cold at $t=0$, there is an initial nonsingular region where $u=0$. Since the reaction is exothermic, the temperature rises till it hits the constraint $T_{\max }$ at time $t=t_{b}$. In the constraint boundary eq. (46) is used. At $t=t_{s}$, the singular region is hit and eq. (45) is used.

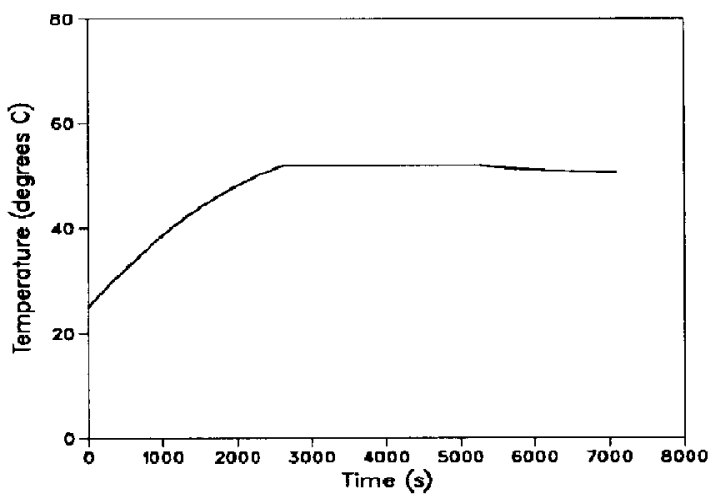

Fig. 1. Optimal temperature profile (example 1).

Table 1. Model parameters

\begin{tabular}{ll}
\hline$k_{10}$ & $1.0 \mathrm{~m}^{3} \mathrm{~kg}^{-1} \mathrm{~s}^{-1}$ \\
$k_{20}$ & $100.0 \mathrm{~s}^{-1}$ \\
$E_{1}$ & $2 \times 10^{4} \mathrm{~J} \mathrm{~mol}^{-1} \mathrm{~K}^{-1}$ \\
$E_{2}$ & $4 \times 10^{4} \mathrm{~J} \mathrm{~mol}^{-1} \mathrm{~K}^{-1}$ \\
$-\Delta H$ & $5 \times 10^{4} \mathrm{~J} \mathrm{~mol}^{-1} \mathrm{~K}^{-1}$ \\
$\rho C_{p}$ & $1000.0 \mathrm{~J} \mathrm{~m}^{-3} \mathrm{~K}^{-1}$ \\
$E$ & $1.5 \times 10^{-5} \mathrm{~kg} \mathrm{~s} \mathrm{~m}^{-3}$ \\
$T_{c}$ & $15 \mathrm{C}$ \\
$T_{\max }$ & $52^{\circ} \mathrm{C}$ \\
\hline
\end{tabular}

Figure 3 shows the profile of the objective function with time. The final time is determined at the point when there is no further decrease in the objective function. Table 3 shows the various switching times as well as the final product concentration. Figure

Table 2. Initial conditions

\begin{tabular}{ll}
\hline$C_{\text {(O) }}$ & $1.0 \mathrm{~kg} \mathrm{~m}^{-3}$ \\
$C_{\text {BO }}$ & $0.0 \mathrm{~kg} \mathrm{~m}^{-3}$ \\
$T(0)$ & $25^{\circ} \mathrm{C}$ \\
\hline
\end{tabular}

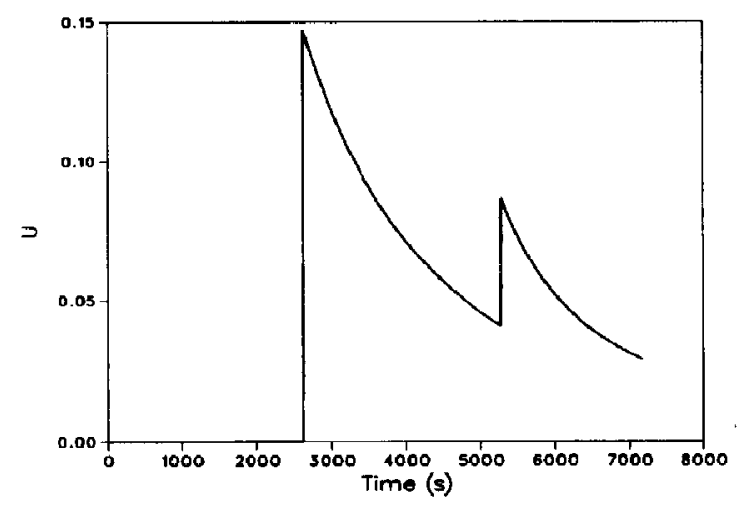

Fig. 2. Optimal cooling rate profile (example 1).

4 shows the time profile of the first-order condition $\lambda^{T} g(x)$ and it is observed that in the singular region $\lambda^{T} g(x)=0$. It was verified (simulation not shown) that indeed $\left(\frac{\partial \phi}{\partial t}+H\right)=0$ at the final time.

The constrained optimization problem was compared with the case where there is no state constraint present. Table 3 shows the results. As expected, the objective function takes a longer time to reach its optimum value when the state constraint is present. The robustness of the control law to errors in the kinetic parameters was tested through numerical simulations. In the control law, the values of the parameters $k_{10}$ and $k_{20}$ used were $10 \%$ lower than 


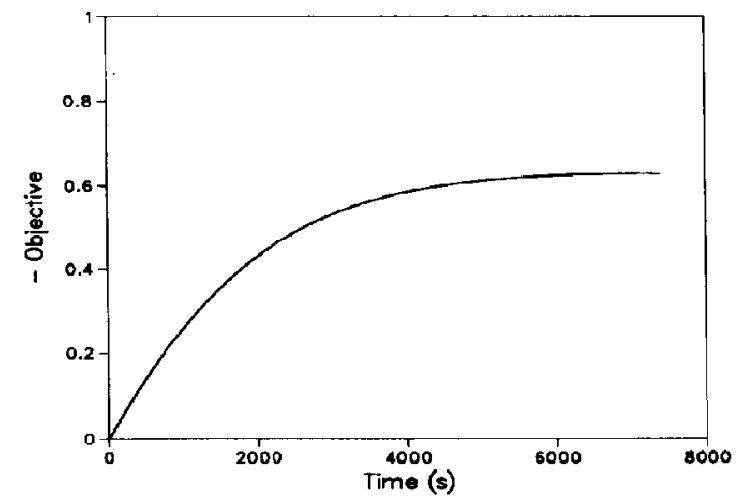

Fig. 3. Profile of objective function (example 1).

Table 3. Constrained vs unconstrained optimization

(Constrained optimization)

Switching time to constrained arc, $2630 \mathrm{~s}$

Switching time to singular arc, $5280 \mathrm{~s}$

Final time, $7170 \mathrm{~s}$

Objective function at final time, $-0.629 \mathrm{~kg} \mathrm{~m}^{-3}$

(Unconstrained optimization)

Switching time to singular arc, $4630 \mathrm{~s}$

Final time, $6750 \mathrm{~s}$

Objective function at final time, $-0.629 \mathrm{~kg} \mathrm{~m}^{-3}$

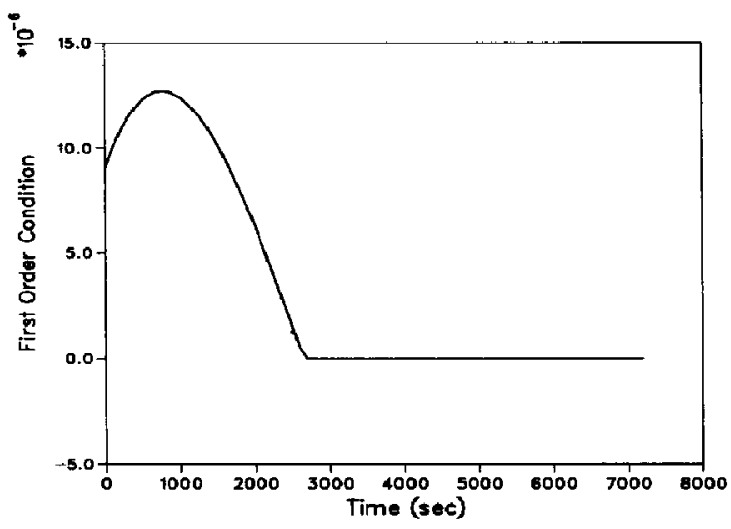

Fig. 4. First-order condition (example 1). those of the system parameters shown in Table 1 . The results are summarized in Table 4 . It is observed that the performance index is very close to that obtained in the nominal case, where the true values of the parameters were used in the control law. Similar simulations can be performed by perturbing other parameter values to define a region in the parameter space where the feedback implementation is successful with the a priori determined switching times.

Example 2: We consider a yield optimization problem in a semi-batch chemical reactor where the following reactions are occurring:

$$
\begin{aligned}
& \text { A } \rightarrow \text { B } \\
& \text { B } \rightarrow \text { C } \\
& \text { A } \rightarrow \text { D. }
\end{aligned}
$$

The reaction from A to $B$ follows the following kinetics:

$$
-r=\frac{k_{1} C_{A} C_{B}}{k_{2}+k_{3} C_{A}+k_{4} C_{A}^{2}} .
$$

All other reactions are first-order reactions. The unsteady state mass balance equations are given by

$$
\begin{aligned}
& \frac{\mathrm{d}\left(C_{A} V\right)}{\mathrm{d} t}=-\frac{k_{1} C_{A} C_{B} V}{k_{2}+k_{3} C_{A}+k_{4} C_{A}^{2}}-k_{D} C_{A} V+C_{A F} u \\
& \frac{\mathrm{d}\left(C_{B} V\right)}{\mathrm{d} t}-\frac{k_{1} C_{A} C_{B} V}{k_{2}+k_{3} C_{A}+k_{4} C_{A}^{2}}-k_{C} C_{B} V \\
& \frac{\mathrm{d}\left(C_{D} V\right)}{\mathrm{d} t}=k_{D} C_{A} V \\
& \frac{\mathrm{d} V}{\mathrm{~d} t}=u .
\end{aligned}
$$

Furthermore, there is a scalar inequality constraint

$$
V-V_{\max } \leqslant 0 \text {. }
$$

We will consider two different objective functions.

Case I: (Objective function independent of the final time) The objective function to be minimized at a free final time is

$$
J=-\left.C_{B} V\right|_{t=t_{s}}
$$

Case II: (Objective function dependent on the final time) The objective function to be minimized at a free

Table 4. Robustness analysis

\begin{tabular}{lll}
\hline Parameters in control law & Parameters in process & Performance index \\
\hline $\begin{array}{l}k_{10}=1.0, k_{20}=100.0 \\
\text { All other parameter } \\
\text { values from Table } 1\end{array}$ & $\begin{array}{l}k_{10}=1.0, k_{20}=100.0 \\
\text { All other parameter } \\
\text { values from Table } 1\end{array}$ & $-0.6296 \mathrm{~kg} \mathrm{~m}^{-3}$ \\
$\begin{array}{l}k_{10}=0.9, k_{20}=90.0 \\
\begin{array}{l}\text { All other parameter } \\
\text { values from Table } 1\end{array}\end{array}$ & $\begin{array}{l}k_{10}=1.0, k_{20}=100.0 \\
\text { All other parameter } \\
\text { values from Table 1 }\end{array}$ & $-0.6295 \mathrm{~kg} \mathrm{~m}^{-3}$ \\
\hline
\end{tabular}


final time is

$$
J=-\left[C_{B} V-e t_{f}\right]
$$

Define

$$
\begin{aligned}
& x_{1}=C_{A} V \\
& x_{2}=C_{B} V \\
& x_{3}=C_{D} V \\
& x_{4}=V .
\end{aligned}
$$

The model can be put in the form

$$
\begin{aligned}
\frac{\mathrm{d}}{\mathrm{d} t}\left[\begin{array}{l}
x_{1} \\
x_{2} \\
x_{3} \\
x_{4}
\end{array}\right]= & {\left[\begin{array}{c}
-\frac{k_{1} x_{1} x_{2} x_{4}}{k_{2} x_{4}^{2}+k_{3} x_{1} x_{4}+k_{4} x_{1}^{2}}-k_{D} x_{1} \\
\frac{k_{1} x_{1} x_{2} x_{4}}{k_{2} x_{4}^{2}+k_{3} x_{1} x_{4}+k_{4} x_{1}^{2}}-k_{C} x_{2} \\
k_{D} x_{1} \\
0
\end{array}\right] } \\
& +\left[\begin{array}{c}
C_{A F} \\
0 \\
0 \\
1
\end{array}\right] u .
\end{aligned}
$$

The system model is of the form

$$
\dot{x}=f(x)+g(x) u
$$

where

$$
f(x)=\left[\begin{array}{c}
x=\left[\begin{array}{l}
x_{1} \\
x_{2} \\
x_{3} \\
x_{4}
\end{array}\right] \\
\frac{k_{1} x_{1} x_{2} x_{4}}{k_{2} x_{4}^{2}+k_{3} x_{1} x_{4}+k_{4} x_{1}^{2}}-k_{C} x_{2} \\
k_{D} x_{1} \\
0 \\
k_{1} x_{1} x_{2} x_{4}+k_{3} x_{1} x_{4}+k_{4} x_{1}^{2} \\
g(x)=\left[\begin{array}{c}
x_{1} x_{1} \\
C_{A F} \\
0 \\
0 \\
1
\end{array}\right] .
\end{array}\right.
$$

It can be easily shown that for this system $n=4$ and $s=1$.

Solution for case I: It is observed that $\phi, f, g$ are independent of $t$. Furthermore, $f(x), g(x), a d_{f}^{1} g(x)$ and $a d_{f}^{2} g(x)$ are linearly independent. Thus, from eq. (27), the optimal static state feedback is given by

$$
u=-\frac{\operatorname{det}\left[f(x) \vdots g(x) \vdots a d_{f}^{1} g(x) \vdots a d_{f}^{2} g(x)\right]}{\operatorname{det}\left[f(x) \vdots g(x) ; a d_{f}^{1} g(x) \vdots\left[g ; a d_{f}^{1} g\right](x)\right]}
$$

Since the reactor starts with a high concentration of $A$ and the reaction rate is decreasing function of $A$ we expect that there will be an initial nonsingular phase which involves feeding of species A. An initial estimate of the switching time was found by performing a one dimensional numerical search as described by $\operatorname{Lim}$ et al. (1986). This estimate was further refined till the first-order condition $\lambda^{T} g(x)=0$ was satisfied on the singular region. When the volume constraint is hit, $u=0$. The final time, $t_{f}$, is determined when the objective function, $\phi$ reaches its optimum value.

Figures 5 and 6 show the optimal input profile and the profile of the objective function for the system parameters in Table 5 and the initial conditions in Table 6. The various switching times and the objective function at the final time are shown in Table 7. Figures 7 and 8 depict the corresponding time profiles of the switching function $\left(\lambda^{T} g(x)\right)$ and the second-order

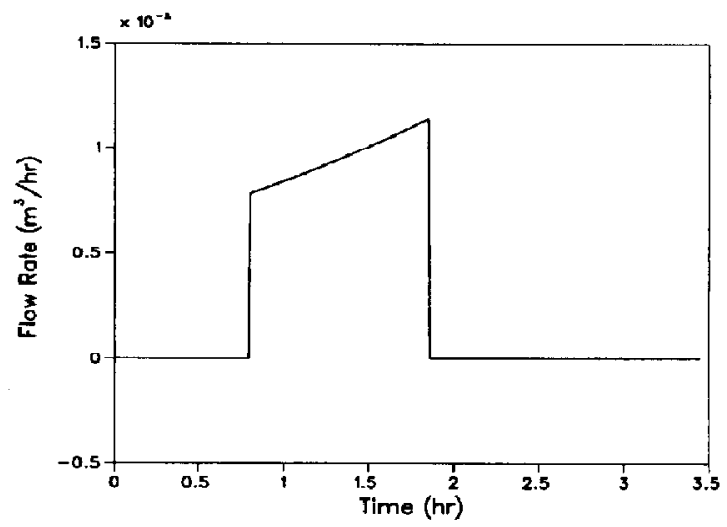

Fig. 5. Optimal input profile (example 2, case I).

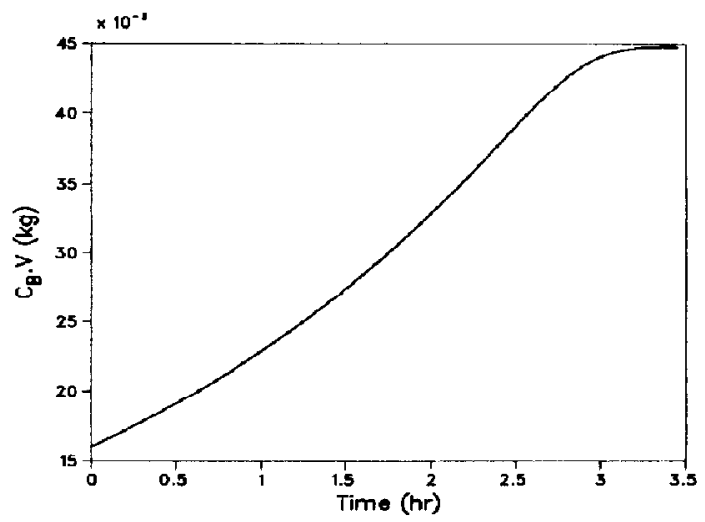

Fig. 6. Profile of objective function (example 2, case I) 
Table 5. Model parameters

\begin{tabular}{ll}
\hline$k_{1}$ & $0.530 \mathrm{~m}^{3} \mathrm{kmol}^{-1} \mathrm{~h}^{-1}$ \\
$k_{2}$ & 1.00 \\
$k_{3}$ & $1.00 \mathrm{~m}^{3} \mathrm{kmol}^{-1}$ \\
$k_{4}$ & $1 / 22 \mathrm{~m}^{3} \mathrm{kmol}^{-2}$ \\
$k_{C}$ & $0.01 \mathrm{~h}^{-1}$ \\
$k_{D}$ & $0.01 \mathrm{~h}^{-1}$ \\
$\varepsilon$ & 2.0 \\
\hline
\end{tabular}

Table 6. Initial conditions

\begin{tabular}{ll}
\hline$C_{A}(0)$ & $7.5 \mathrm{~kg} \mathrm{~m}^{-3}$ \\
$C_{B}(0)$ & $8.0 \mathrm{~kg} \mathrm{~m}^{-3}$ \\
$C_{D}(0)$ & $0.0 \mathrm{~kg} \mathrm{~m}^{-3}$ \\
$V(0)$ & $2.0 \times 10^{-3} \mathrm{~m}^{3}$ \\
\hline
\end{tabular}

Table 7. Constrained optimization (example 2, case I)

Switching time to singular arc, $0.793 \mathrm{~h}$

Switching time to constrained arc, $1.86 \mathrm{~h}$

Final time, $3.39 \mathrm{~h}$

Objective function at final time; $-44.810 \times 10^{-3} \mathrm{~kg}$

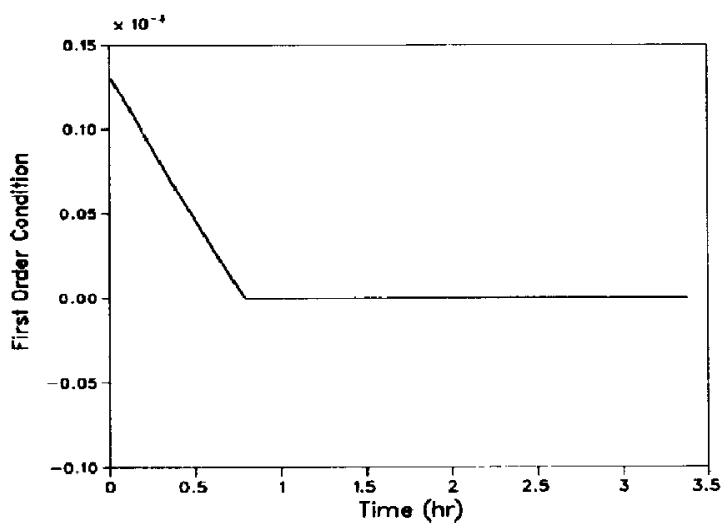

Fig. 7. First-order condition (example 2, case I).

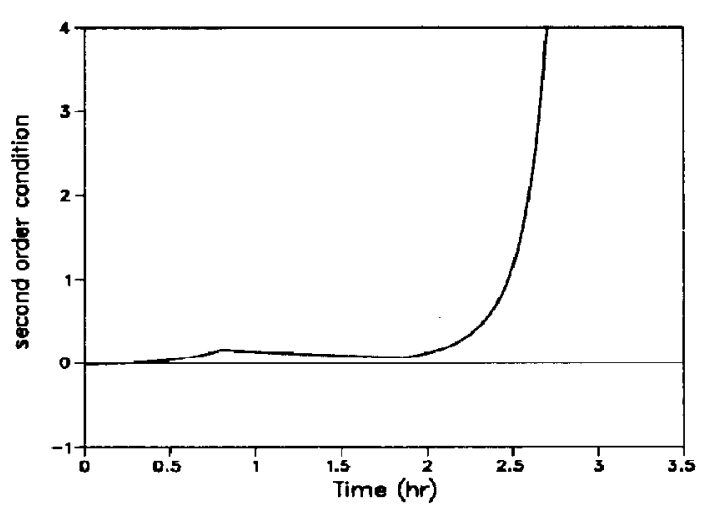

Fig. 8. Second-order condition (example 2, case I). condition $\left(-\lambda^{T}\left[g, a d_{j}^{1} g\right](x)\right)$. The switching function vanishes on the singular time interval and is positive everywhere else. The positivity of the switching function is consistent with $u(t)$ taking its minimum value $(u=0)$ in the nonsingular interval. Furthermore, the second-order condition $-\lambda^{T}\left[g, a d_{f}^{1} g\right](x, t) \geqslant 0$ is satisfied in the singular region and so the singular extremal is indeed optimal. The final time, $t_{f}$, is determined when the objective function $\phi$ reaches its optimum value. It was verified (simulation not shown) that indeed $\boldsymbol{H}=\mathbf{0}$ everywhere.

The robustness of the state feedback law with respect to errors in the system parameter $k_{1}$ was tested through numerical simulations. With the switching time from the nominal case, the static feedback law was implemented using a $13.4 \%$ higher $k_{1}$ than its true value. The results are summarized in Table 8 . It is observed that the performance index is very close to that obtained in the nominal case. Similar simulations can be performed, by perturbing other parameter values, to obtain a region in parameter space where the feedback implementation is successful with the a priori determined switching times.

The sensitivity of the performance index to errors in the switching time to the singular region was checked. Assuming various values of the switching time to the singular region, the feeding profile was calculated using the static state feedback law in the singular region. When the volume constraint was hit, $u$ was set to zero. The final time $t_{f}$ was determined when the objective function $\phi$ reached its optimum value. The results are shown in Table 9 . It is observed that the final performance index is quite robust to errors in the assumed switching time. From this study we conclude that the performance index is not very sensitive to errors in the switching time and so an a priori estimate of the switching time can be used in closed-loop implementation.

Solution for case II: It can be easily verified that the vector fields $g(x), \operatorname{ad}_{f}^{1} g(x), a d_{f}^{2} g(x), a d_{f}^{3} g(x)$ are linearly independent. Thus the optimal state feedback in the singular region will be dynamic. Substituting $n=4$ and $s=1$ in eq. (28) we obtain a dynamic feedback law by setting the determinant of

$$
\begin{aligned}
& g(x) \\
& a d_{f}^{1} g(x) \\
& a d_{f}^{2} g(x)+\left[g, a d_{f}^{1} g\right](x) u \\
& a d_{f}^{3} g(x)+\left(\left[g, a d_{f}^{2} g\right](x)+\left[f,\left[g, a d_{f}^{1} g\right]\right](x)\right. \\
& \quad+\left[g,\left[g, a d_{f}^{1} g\right](x) u\right) u+\left[g, a d_{f}^{1} g\right](x) \frac{d u}{d t}
\end{aligned}
$$

equal to zero.

Figures 9 and 10 show the optimal input profile and the profile of the objective function for the system parameters in Table 5 and the initial conditions in Table 6 . The various switching times and the objective function at the final time are shown in Table 10. Figures 11 and 12 depict the corresponding time pro- 
Table 8. Robustness analysis

\begin{tabular}{|c|c|c|c|}
\hline Parameters in control law & Parameters in process & Performance index & Final time \\
\hline $\begin{array}{l}k_{1}=0.530 \\
\text { All other parameter } \\
\text { values from Table } 5\end{array}$ & $\begin{array}{l}k_{1}=0.530 \\
\text { Alt other parameter } \\
\text { values from Table } 5\end{array}$ & $-44.810 \times 10^{-3} \mathbf{k g}$ & $3.39 h^{-1}$ \\
\hline $\begin{array}{l}k_{1}=0.600 \\
\text { All other parameter } \\
\text { values from Table } 5\end{array}$ & $\begin{array}{l}k_{1}=0.530 \\
\text { All other parameter } \\
\text { values from Table } 5\end{array}$ & $-44.718 \times 10^{-3} \mathrm{~kg}$ & $3.39 h^{-1}$ \\
\hline
\end{tabular}

Table 9. Sensitivity of error in switching time on performance index

\begin{tabular}{ccc}
\hline $\begin{array}{c}\text { Assumed switching } \\
\text { time (h) }\end{array}$ & $\begin{array}{c}\text { Performance } \\
\text { index (kg) }\end{array}$ & $\begin{array}{c}\text { Final } \\
\text { time (h) }\end{array}$ \\
\hline 0.75 & $-44.676 \times 10^{-3}$ & 3.31 \\
0.791 & $-44.810 \times 10^{-3}$ & 3.39 \\
0.85 & $-44.760 \times 10^{-3}$ & 3.39 \\
0.90 & $-44.720 \times 10^{-3}$ & 3.39 \\
\hline
\end{tabular}

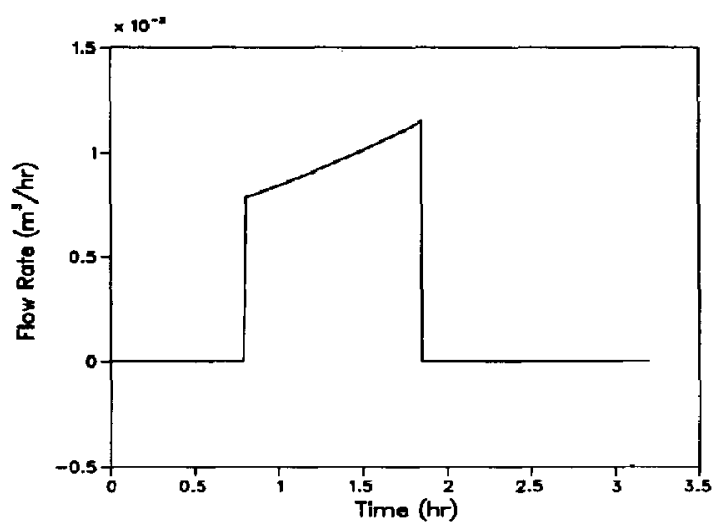

Fig. 9. Optical input profile (example 2, case II).

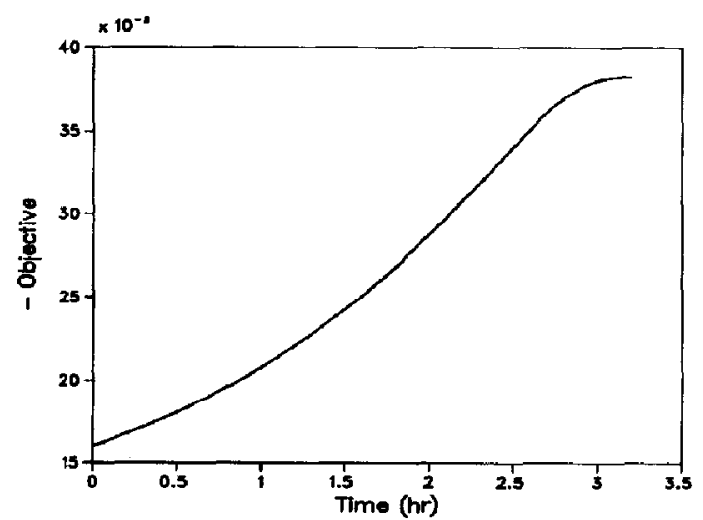

Fig. 10. Profile of objective function (example 2, case II).
Table 10. Constrained optimization (example 2, case II)

Switching time to singular arc, $0.793 \mathrm{~h}$

Switching time to constrained arc, $1.86 \mathrm{~h}$

Final time, $3.19 \mathrm{~h}$

Objective function at final time, $-38.3 \times 10^{-3} \mathrm{~kg}$

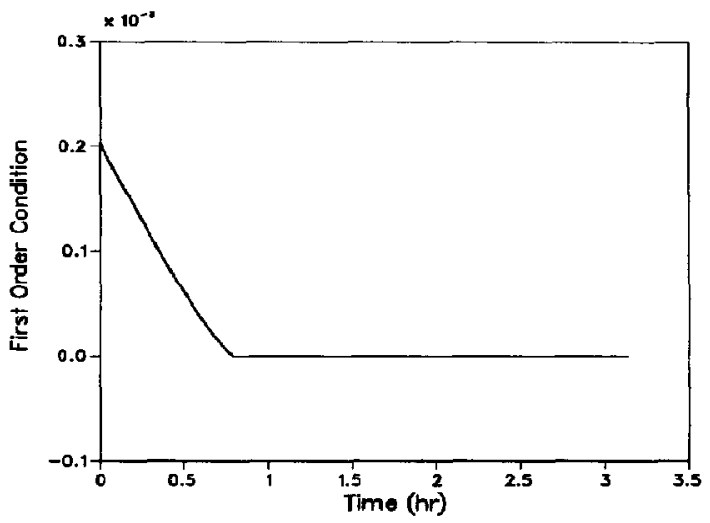

Fig. 11. First-order condition (example 2, case II).

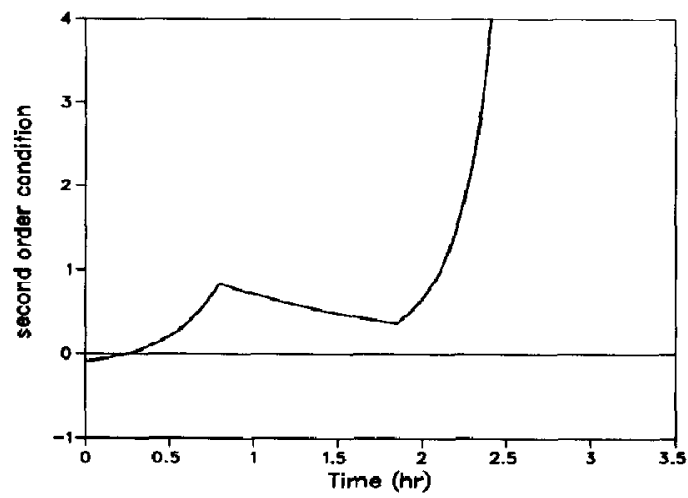

Fig. 12. Second-order condition (example 2, case II).

Table 11. Sensitivity of error in initial condition of $u(t)$ on performance index

\begin{tabular}{cc}
\hline $\begin{array}{c}\text { Assumed initial } \\
\text { condition of } u(t)\end{array}$ & $\begin{array}{c}\text { Performance } \\
\text { index }(\mathrm{kg})\end{array}$ \\
\hline 0.60 & $-38.3 \times 10^{-3}$ \\
0.75 & $-38.3 \times 10^{-3}$ \\
0.90 & $-38.3 \times 10^{-3}$ \\
\hline
\end{tabular}


Table 12. Robustness analysis

\begin{tabular}{|c|c|c|}
\hline Parameters in control law & Parameters in process & Performance index \\
\hline $\begin{array}{l}k_{1}=0.530 \\
\text { All other parameter } \\
\text { values from Table } 5\end{array}$ & $\begin{array}{l}k_{1}=0.530 \\
\text { All other parameter } \\
\text { values from Table } 5\end{array}$ & $-38.3 \times 10^{-3} \mathrm{~kg}$ \\
\hline $\begin{array}{l}k_{1}=0.56 \\
\text { All other parameter } \\
\text { values from Table } 5\end{array}$ & $\begin{array}{l}k_{1}=0.530 \\
\text { All other parameter } \\
\text { values from Table } 5\end{array}$ & $-38.3 \times 10^{-3} \mathrm{~kg}$ \\
\hline $\begin{array}{l}k_{1}=0.59 \\
\text { All other parameter } \\
\text { values from Table } 5\end{array}$ & $\begin{array}{l}k_{1}=0.530 \\
\text { All other parameter } \\
\text { values from Table } 5\end{array}$ & $-38.3 \times 10^{-3} \mathbf{k g}$ \\
\hline
\end{tabular}

files of the switching function $\left(\lambda^{T} g(x)\right)$ and the secondorder condition $\left(-\lambda^{T}\left[g, a d_{f}^{1} g\right](x)\right)$. The switching function vanishes on the singular time interval and is positive everywhere else. The positivity of the switching function is consistent with $u(t)$ taking its minimum value $(u=0)$ in the nonsingular interval. Furthermore, the second-order condition $-\lambda^{T}\left[g, a d_{f}^{1} g\right](x, t) \geqslant 0$ is satisfied in the singular rcgion and so the singular extremal is indeed optimal. The final time $t_{f}$ is determined when the objective function $\phi$ reaches its optimum value. It was verified (simulation not shown) that indeed $\left(\frac{\partial \phi}{\partial t}+H\right)=0$ at the final time.

Since the optimal state feedback is dynamic, we need the initial condition of $u$ to implement the state feedback law. The effect of error in the initial condition of $u$ on the final performance index was studied. The results are shown in Table 11 . It is observed that the final performance index is very robust to errors in the assumed initial condition for $u(t)$.

From this study we conclude that the performance index is not sensitive at all to errors in the initial condition for $u(t)$ in the range of initial conditions considered and so an a priori estimate of the initial condition for $u(t)$ can be used in closed-loop implementation.

The robustness of the dynamic state feedback law with respect to errors in the system parameter $k_{1}$ was tested. The results are summarized in Table 12 . It is observed that the performance index is very close to that obtained in the nominal case. Similar simulations can be performed by perturbing other parameter values to determine the region in parameter space where the feedback implementation is successful.

Acknowledgement-Financial support by NSF Research Grant BCS-8912627 is gratefully acknowledged.

\section{NOTATION}

$C_{A}$ concentration of species $\mathrm{A}, \mathrm{kg} \mathrm{m}^{-3}$

$C_{A F} \quad$ concentration of species $A$ in feed, $\mathrm{kg} \mathrm{m}^{-3}$

$C_{B} \quad$ concentration of species $B, \mathrm{~kg} \mathrm{~m}^{-3}$

$C_{C} \quad$ concentration of species $\mathrm{C}, \mathrm{kg} \mathrm{m}^{-3}$

$C_{D}$ concentration of species $\mathrm{D}, \mathrm{kg} \mathrm{m}^{-3}$
$C_{p} \quad$ specific heat, $\mathrm{J} \mathrm{kg}^{-1} \mathrm{~K}^{-1}$

$E_{1}, E_{2}$ activation energies, $J_{\mathrm{mol}^{-1}} \mathrm{~K}^{-1}$

$H$ Hamiltonian

$H_{w} \quad$ first derivative of Hamiltonian with respect to $u$

$H_{u u} \quad$ second derivative of Hamiltonian with respect to $u$

$l \quad$ identity matrix

$J$ performance index

$k_{B} \quad$ kinetic rate constant of reaction $A \rightarrow B, h^{-1}$

$L_{f}^{k} C(x) k$ th Lie derivative of $f(x)$ and $C(x)$

$T$ temperature, $K$

$V \quad$ volume, $\mathrm{m}^{3}$

$\operatorname{ad}_{f}^{1} g(x)$ Lie bracket $\{[f, g](x)\}$

$a d_{f}^{k} g(x)$ Lie bracket of order $k\left\{\left[f, a d_{f}^{k}{ }^{1} g\right](x)\right\}$

det determinant

$f \quad$ vector function in dynamic model

$g$ vector function in dynamic model

$k_{1} \quad$ kinetic rate constant

$k_{2} \quad$ kinetic rate constant

$k_{3} \quad$ kinetic rate constant

$k_{4} \quad$ kinetic rate constant

$k_{C} \quad$ kinetic rate constant of reaction $\mathrm{B} \rightarrow \mathrm{C}, \mathrm{h}^{-1}$

$k_{\mathrm{D}} \quad$ kinetic rate constant of reaction $\mathrm{A} \rightarrow \mathrm{D}, \mathrm{h}^{-1}$

$n$ number of state equations (dimension of state vector $x$ )

degree of singularity

time

final time

manipulated input

vector of system states

output

Greek symbols

$\beta \quad$ adjustable parameter

$\lambda$ vector of adjoint states

$\phi \quad$ scalar function expressing the performance index in terms of the system states

$\rho$ density, $\mathrm{kg} \mathrm{m}^{-3}$

\section{REFERENCES}

Bryson, A. E., Denham, W. F. and Dreyfus, S. E., 1963, Optimal programming problems with inequality constraints 1: necessary conditions for extremal solutions. AIAA J. I(11), 2544. 
Bryson, A. E. and Ho, Y. C., 1975, Applied Optimal Control. Wiley, New York.

Gabasov, R. and Kirillova, F. M., 1972, Higher order necessary conditions for optimality. SIAM J. Control $10(1), 127$.

Hunt, L. R., 1982, Sufficient conditions for controllability. IEEE Trans. Circuits Systems CAS-29(5), 285.

Krener, A. J., 1977, The higher order maximal principle and its application to singular extremals. SIAM J. Control Optimization 15(2), 256

Modak, J. M., 1988, A theoretical and experimental optimization of fed-batch fermentation processes. Ph.D. thesis, Purdue University, West Lafayette. IN.

Kravaris, C. and Chung. C. B., 1987, Nonlinear state feedback synthesis by global input output linearization. A.I.Ch.E. J. 33(4), 592.

Lim, H. C., Modak, J. M. and Bonte, P., 1986, Computational algorithms for optimal feed rates for a class of fed-batch fermentation: numerical results for penicillin and cell mass production. Biotechnol. Bioengng 28, 1408.

Maurer, H., 1977, On optimal control problems with bounded state variables and control appearing linearly. SIAM J. Control Optimization 15(3), 345.

Palanki, S., Kravaris, C. and Wang, H. Y., 1993, Synthesis of state feedback laws for end-point optimization in batch processes. Chem. Engng Sci. 48(1), 135.

Speyer, J. L., and Bryson, A. E., 1968, Optimal programming problems with a bounded state space. $A I A A J$. G(8), 1488.

\section{APPENDIX A: PROOF OF THEOREM}

The first-order necessary conditions on the singular extremal are as follows:

$$
\begin{aligned}
\lambda^{T} g(x) & =0 \\
\lambda^{T} a d_{f}^{1} g(x) & =0 \\
\lambda^{T} a d_{f}^{2} g(x) & =0 \\
\vdots & \\
\dot{\lambda}^{T} a d_{f}^{n-2} g(x) & =0 \\
\dot{\lambda}^{T} a d_{f}^{n-1} g(x) & =0 .
\end{aligned}
$$

Eliminating the $n$-vector of adjoint states, we obtain the singular surface (24) on which the state trajectories corresponding to the singular extremal evolve.

\section{APPENDIX B: PROOF OF THEOREM 2}

Proof for (i): The first-order necessary conditions are given by

$$
\begin{aligned}
\lambda^{T} f(x) & =0 \\
\lambda^{T} g(x) & =0 \\
\lambda^{T} a d_{f}^{1} g(x) & =0 \\
\lambda^{T} a d_{f}^{2} g(x) & =0 \\
\vdots & \\
\lambda^{T} a d_{f}^{n-2} g(x) & =0 .
\end{aligned}
$$

Eliminating the $n$ adjoint states from the above $n$ independent equations, we obtain the singular surface (25) on which the state tajectories corresponding to the singular extremal evolve.

Proof for (ii): The first-order necessary conditions are given by

$$
\begin{gathered}
\lambda^{T} g(x)=0 \\
\lambda^{T} a d_{f}^{1} g(x)=0 \\
\vdots \\
\lambda^{T} a d_{f}^{n-2} g(x)=0 \\
\lambda^{T} a d_{f}^{-1} g(x)+\lambda^{T}\left[g, a d_{f}^{n-2} g\right](x) u=0 .
\end{gathered}
$$

Since these are $n$ equations in $n$ adjoint states, we can eliminate the adjoint states $\lambda^{T}=\left[\lambda_{1} \lambda_{2} \ldots \lambda_{n}\right]$ to obtain the static feedback represented by eq. (26).

\section{APPENDIX C: PROOF OF THEOREM 3}

Proof for (i): The first-order necessary conditions for $s=n-3$ can be written as

$$
\begin{aligned}
& \lambda^{T} f(x)=0 \\
& \lambda^{T} g(x)=0 \\
& \lambda^{T} \operatorname{ad}_{f}^{1} g(x)=0 \\
& \vdots \\
& \lambda^{T} a d_{f}^{n-3} g(x)=0 \\
& \lambda^{T} a d_{f}^{n-2} g(x)+\lambda^{T}\left[g \cdot a d_{f}^{n-3} g\right](x) u=0 .
\end{aligned}
$$

Since these are $n$ equations in $n$ adjoint states, we can eliminate the adjoint states $\lambda^{T}=\left[\hat{\lambda}_{1}, \hat{\lambda}_{2} \ldots \hat{\lambda}_{n}\right]$ to obtain the static state feedback represented by eq. (27).

Proof for (ii): From proposition 2, the first-order necessary conditions for singular extremal are

$$
\begin{gathered}
\dot{\lambda}^{T} g(x)=0 \\
\lambda^{T} a d_{f}^{\top} g(x)=0 \\
\vdots \\
\lambda^{T} a d_{f}^{n-3} g(x)=0 \\
\lambda^{T} a d_{f}^{n-2} g(x)+\lambda^{T}\left[g, a d_{f}^{n-3} g\right](x) u(t)=0 .
\end{gathered}
$$

which constitute $n-1$ equations in $n$ adjoint states. Differentiating the last equation once we obtain an additional equation

$$
\begin{aligned}
& a d_{f}^{n-1} g(x)+\left[g, a d_{f}^{n-2} g\right](x) u(t) \\
& \quad+\left(I \frac{\mathrm{d}}{\mathrm{d} t}-\frac{\partial f(x)}{\partial x}-\frac{\partial g(x)}{\partial x} u(t)\right)\left(\left[g, a d_{f}^{n-3} g\right](x) u(t)\right) .
\end{aligned}
$$

The above equations constitute a set of $n$ equations in $n$ adjoint variables which can be eliminated to give the dynamic state feedback law. This is obtained by setting the determinant of the vector fields in eq. (29) equal to zero.

\section{APPENDIX D: PROOF OF THEOREM 4}

Proof for (i): The first-order necessary conditions for singular extremal are

$$
\begin{gathered}
\lambda^{T} f(x)=0 \\
\lambda^{T} g(x)=0 \\
\lambda^{T} a d_{f}^{1} g(x)=0 \\
\vdots \\
\lambda^{T} a d_{f}^{s-1} g(x)=0 \\
\lambda^{T} a d_{f}^{s} g(x)+\lambda^{T}\left[g, a d_{f}^{s-1} g\right](x) u(t)=0
\end{gathered}
$$

which constitute $s+2$ equations in $n$ adjoint states. Differentiating the last equation $(n-s-3)$ times we obtain $(n-s-3)$ more equations:

$$
\begin{aligned}
& \lambda^{T}\left(a d_{f}^{s+1} g(x)+\left[g, a d_{f}^{s} g\right](x) u(t)\right. \\
& \left.\quad+\left(I \frac{\mathrm{d}}{\mathrm{d} t}-\frac{\partial f(x)}{\partial x}-\frac{\partial g(x)}{\partial x} u(t)\right)\left(\left[g, a d_{f}^{s-1} g\right](x) u(t)\right)\right)=0 \\
& \lambda^{T}\left(a d_{f}^{s+2}(g(x))+\left[g, a d_{f}^{s+1} g\right](x) u(t)\right. \\
& \quad+\left(I \frac{\mathrm{d}}{\mathrm{d} t}-\frac{\partial f(x)}{\partial x}-\frac{\partial g(x)}{\partial x} u(t)\right)\left(\left[g, a d_{f}^{3} g\right](x) u(t)\right)
\end{aligned}
$$




$$
\begin{aligned}
& +\lambda^{T}\left(1 \frac{\mathrm{d}}{\mathrm{d} t}-\frac{\partial f(x)}{\partial x}-\frac{\partial g(x)}{\partial x} u(t)\right)^{2}\left(\left[g, a d_{f}^{-1} g\right](x) u(t)\right)=0 \\
& \vdots \\
& \lambda^{T}\left(a d_{f}^{n-2} g(x)+\sum_{k=0}^{n-3}\left(I \frac{\mathrm{d}}{\mathrm{d} t}-\frac{\partial f(x)}{\partial x}-\frac{\partial g(x)}{\partial x} u(t)\right)^{k}\right. \\
& \left.\quad \times\left(\left[g, a d_{f}^{n-3} g\right](x) u(t)\right)\right)=0 .
\end{aligned}
$$

The above equations constitute a set of $n$ equations in $n$ adjoint variables which can be eliminated to give the dynamic state feedback law. This is obtained by setting the determinant of the vector fields in eq. (30) equal to zero.

Proof for (ii): The first order necessary conditions for singular extremal are

$$
\begin{gathered}
\dot{\lambda}^{T} g(x)=0 \\
\lambda^{r} a d_{f}^{\prime} g(x)=0 \\
\vdots \\
\lambda^{T} a d_{f}^{s} g(x)=0 \\
\lambda^{T} a d_{f}^{s+1} g(x)+\lambda^{T}\left[g, a d_{f}^{s} g\right](x) u(t)=0 .
\end{gathered}
$$

which constitute $s+2$ equations in $n$ adjoint states. Differentiating the last equation $(n-s-2)$ times we obtain $(n-s-2)$ more equations:

$\lambda^{T}\left(a d_{f}^{3+2} g(x)+\left[g, a d_{f}^{s+1} g\right](x) u(t)\right.$

$+\left(I \frac{\mathrm{d}}{\mathrm{d} t}-\frac{\partial f(x)}{\partial x}-\frac{\partial g(x)}{\partial x} u(t)\right)\left(\left[g, a d_{f}^{s} g\right](x) u(t)\right)=0$

$\lambda^{T}\left(a d_{f}^{s+3}(g(x))+\left[g, a d_{f}^{s+2} g\right](x) u(t)\right.$

$+\left(1 \frac{\mathrm{d}}{\mathrm{d} t}-\frac{\partial f(x)}{\partial x}-\frac{\partial y(x)}{\partial x} u(t)\right)\left(\left[g, a d_{f}^{s+1} g\right](x) u(\ell)\right)$

$+\lambda^{T}\left(I \frac{\mathrm{d}}{\mathrm{d} t}-\frac{\partial f(x)}{\partial x}-\frac{\partial g(x)}{\partial x} u(t)\right)^{2}\left(\left[g, a d_{f}^{x} g\right](x) u(t)\right)=0$

$\lambda^{T}\left(a d_{f}^{n-1} g(x)+\sum_{k=0}^{n-s-2}\left(I \frac{d}{d t}-\frac{\partial f(x)}{\partial x}-\frac{\partial g(x)}{\partial x} u(t)\right)^{k}\right.$

$\left.x\left(\left[g, a d_{f}^{\eta-k-2} q\right](x) u(t)\right)\right)=0$.

The above equations constitute a set of $n$ equations in $n$ adjoint variables which can be eliminated to give the dynamic state feedback law. This is obtained by setting the determinant of the vector fields in eq. (31) equal to zero. 